\title{
ARTICLE \\ Brain structure, cognition, and brain age in schizophrenia, bipolar disorder, and healthy controls
}

\author{
Saba Shahab ${ }^{1,2,3}$, Benoit H. Mulsant ${ }^{2,4,5,6}$, Melissa L. Levesque ${ }^{1,2}$, Navona Calarco ${ }^{1,2,5}$, Arash Nazeri (iD) , Anne L. Wheeler ${ }^{8,9}$, \\ George Foussias $2,4,5,10$, Tarek K. Rajji ${ }^{2,4,5,6}$ and Aristotle N. Voineskos $1,2,4,5,6,10$
}

Schizophrenia and bipolar disorder (BD) may be disorders of accelerated aging. Direct comparison of healthy aging populations with schizophrenia and BD patients across the adult lifespan may help inform this theory. In total, 225 individuals (91 healthy controls, 81 schizophrenia, 53 euthymic BD) underwent 3T T1-weighted magnetic resonance imaging, diffusion tensor imaging, and cognitive testing. We analyzed associations among age, diagnosis, and cognition with cortical thickness and fractional anisotropy (FA) using general linear models. We then assessed "brain age" using a random forest algorithm, which was also assessed in an independent sample $(n=147)$. Participants with schizophrenia had lower cortical thickness and FA compared with the other two groups, most prominently in fronto-temporal circuitry. These brain changes were more evident in younger participants than in older ones, yet were associated with cognitive performance independent of diagnosis. Predicted age was 8 years greater than chronological age in individuals with schizophrenia in the first sample and 6 years greater in the second sample. Predicted and chronological age were not different in BD. Differences in brain circuitry are present from illness onset most prominently in schizophrenia and to a lesser extent in BD. These results support a non-progressive "early hit" hypothesis/etiology of illness in the major psychoses. Brain age differences support the hypothesized early aging mechanism in schizophrenia but not in BD.

Neuropsychopharmacology (2019) 44:898-906; https://doi.org/10.1038/s41386-018-0298-z

\section{INTRODUCTION}

Converging evidence has accumulated suggesting that schizophrenia and bipolar disorder (BD) may be disorders associated with accelerated aging [1-3]. Individuals with schizophrenia and $\mathrm{BD}$ have shortened life spans compared with healthy controls (HC) $[4,5]$. In the brain, alterations found in schizophrenia and $\mathrm{BD}$ compared with $\mathrm{HCs}$ are very similar to those observed in healthy aging [6-8]. Specifically, aging is associated with decreased cortical thickness throughout the brain $[9,10]$, but most notably in frontal and temporal cortices [11]. Likewise, widespread reduction in cortical thickness, particularly in frontotemporal areas, is found in schizophrenia and BD compared with $\mathrm{HCs}[5,7,8]$. There is also a considerable literature on fractional anisotropy (FA) changes in healthy aging [12-14]. Our group, studying individuals $18-85$ years of age, has found that younger people with schizophrenia have low FA, similar to older people with schizophrenia and older HCs, whereas younger HCs have high FA [12]. Data from another group, using a different approach in a cross-sectional sample, showed that aging individuals with schizophrenia lose frontal white matter integrity twice as fast as HCs [15]; however this study and most others exploring age-related change in the major psychoses do not typically include individuals in the final decades of the adult lifespan.

Neural alterations in schizophrenia and BD appear to be functionally relevant. Much work supports an association between cognitive function and neurobiological alterations, particularly to white matter [16-18]. Cognitive impairments in individuals with schizophrenia and BD are similar to those seen in older controls $[5,19]$. Some studies suggest that the decline in cognitive function with age may be accelerated in psychiatric disorders including schizophrenia [20] and BD [5]. Alternatively, Fears and colleagues have shown that the association between cognitive performance and age is similar across diagnoses for some specific functions but differs across diagnoses for others. Therefore, the relationships among brain structure, cognitive performance, serious mental illness, and aging require further disentanglement. Cognitive variability has been shown to be captured by a single factor in both schizophrenia and BD [21, 22]. There also remains some debate regarding altered neurodevelopmental versus (early) neurodegenerative trajectories of the major psychoses (i.e., "an early hit" versus an accelerated aging mechanism), especially in schizophrenia. An early hit non-progressive mechanism would involve an initial change in cognitive performance and brain structure that either stays the same throughout the remaining life

\footnotetext{
${ }^{1}$ Kimel Family Translational Imaging-Genetics Laboratory, Research Imaging Centre, Centre for Addiction and Mental Health, Toronto, ON, Canada; ${ }^{2}$ Campbell Family Mental

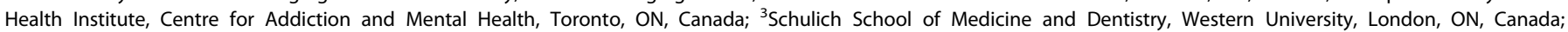

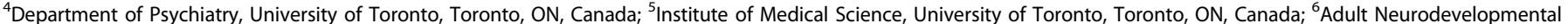

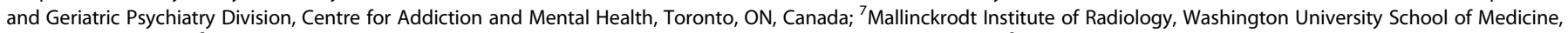

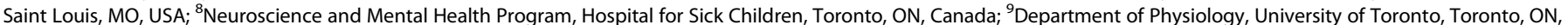
Canada and ${ }^{10}$ Schizophrenia Division, Centre for Addiction and Mental Health, Toronto, ON, Canada

Correspondence: Aristotle N. Voineskos (Aristotle.Voineskos@camh.ca)
}

Received: 31 July 2018 Revised: 9 December 2018 Accepted: 13 December 2018

Published online: 20 December 2018 
of an individual or changes at the same rate that the measure changes in a healthy individual. Conversely, accelerated aging would mean that the cognitive performance and structural changes are worsening at a rate that is significantly higher than the rate at which these measures are changing in a healthy population. Differences in older adults would be reduced or stay the same as the differences in younger adults if the first mechanism were in play. If accelerated aging was observed, differences in brain structure and cognitive performance would be larger in the older adult groups than the younger groups.

\section{OBJECTIVE}

Our objectives were to: (1) investigate differences in brain structure (cortical thickness and white matter $F A$ ) in relation to age and diagnosis; (2) assess the association between cognitive performance and brain structure across $\mathrm{HCs}$ and individuals with schizophrenia and BD; and (3) compare the difference between predicted and chronological age in the same three groups. We hypothesized that cortical thickness and FA would be lower in fronto-temporal regions in both individuals with schizophrenia and BD compared with HCs. Cognitive deficits in BD have been shown to be less prominent than those seen in schizophrenia $[23,24]$, thus we expect that BD would demonstrate a neural phenotype intermediate between HCs and schizophrenia. We also expected a significant relationship between brain structure and cognitive performance independent of diagnosis. Finally, we hypothesized that the difference between brain age and chronological age would be greater in individuals with schizophrenia and BD than in HCs.

\section{MATERIALS AND METHODS}

Participants

Two samples were recruited. The first sample comprised 225 participants: $91 \mathrm{HCs}, 81$ clinically stable outpatients with a schizophrenia spectrum disorder (SSD, i.e. schizophrenia, schizoaffective disorder, schizophreniform disorder, or psychotic disorder not otherwise specified), and 53 euthymic outpatients with bipolar disorder (45 BD I, 8 BD II), both with and without a history of psychosis when ill, across the adult lifespan (1883 years) who were recruited and underwent clinical assessments and $3 \mathrm{~T}$ neuroimaging at the Centre for Addiction and Mental Health (CAMH) in Toronto, Canada. The second sample comprised 147 participants (80 HCs and 67 schizophrenia outpatients) who were recruited and underwent clinical assessments at $\mathrm{CAMH}$ and 1.5T neuroimaging at Toronto General Hospital, Toronto, Canada. Exclusion criteria for both samples were intelligence quotient $<70$ as estimated by the Wechsler Test for Adult Reading (WTAR) [25], substance dependence or abuse reported or indicated by a urine toxicology screen, head trauma with loss of consciousness, neurological disorders, and any magnetic resonance imaging contraindications. Individuals with schizophrenia were clinically stable as determined by no medication change within the past month, and individuals with BD were determined as euthymic based on scores on the Hamilton Depression Rating Scale (HAM-D) [26] by a score of $\leq 17$ and Young Mania Rating Scale (YMRS) [27] by a score of $\leq 10$. A first degree relative with a primary psychotic disorder was also an exclusion criterion for HCs. The study protocols were approved by the appropriate ethics committees and all participants provided written informed consent.

Demographic and clinical measures

Socio-demographic information was collected for all participants including age, sex, handedness, years of education, and parental education. All participants completed the Structured Clinical Interview for DSM-IV-TR Axis I Disorders [28]. Participants with schizophrenia and BD were assessed for age of onset, medication use, and symptom severity. Age of onset and medication use were self-reported and verified using medical records when necessary, and antipsychotic daily doses were converted to chlorpromazine equivalents. Symptom severity was characterized by either the Positive and Negative Syndrome Scale (PANSS) [29] or Brief Psychiatric Rating Scale (BPRS) [30] in participants with schizophrenia (those who received the BPRS also received the Scale for the Assessment of Negative Symptoms (SANS) [31]), and in participants with BD using the HAM-D and YMRS.

\section{Neurocognitive assessment}

The first sample underwent cognitive testing using assessments from the MATRICS Consensus Cognitive Battery [32, 33]. We did not test participants on the MATRICS' MSCEIT, as participants were tested on a more extensive social cognitive battery (not analyzed here). In addition, we tested participants on Trails Making Test: Part B [34] to enhance assessment of executive function.

Image acquisition

In the first sample, structural and diffusion images were acquired on a 3T Discovery MR750 system (General Electric, Milwaukee, WI) with an eight-channel head coil. In the first sample, T1-weighted anatomical images were acquired using a 3D inversion-prepared fast spoiled gradient-recalled echo acquisition, FSPGR-BRAVO (TE/ $\mathrm{TR}=3 / 6.7 \mathrm{~ms}$ ) with an isotropic voxel size of $0.9 \mathrm{~mm}^{3}$. Diffusionweighted data were acquired using an echo planar sequence with diffusion gradients applied in 60 non-collinear directions and $b=$ $1000 \mathrm{~s} / \mathrm{mm}^{2}$. Five b0 images were obtained. Additional protocol details including image acquisition for the second sample are available in the Supplementary section.

Image analysis

For both samples, T1-weighted images were submitted to the CIVET pipeline (version 1.1.10, Montreal Neurological Institute) to generate cortical thickness models [35], and the R package "mni. cortical.statistics" (Brain Imaging Centre, Montreal Neurological Institute) was used for cortical thickness analyses. Diffusion images were preprocessed using FMRIB's Software Library (FSL, www.fmrib.ox.ac.uk) and were then submitted to FSL's TBSS and non-parametric permutation-based analysis Randomize [36]. For the age prediction analysis, averaged cortical thickness and FA values were extracted for regions of interest. The AAL atlas was used to obtain averaged cortical thickness for 78 brain regions [37] and the ENIGMA-DTI pipeline (http://enigma.ini.usc.edu/ongoing/ dti-working-group/) [38] was used to obtain averaged FA for 38 regions defined in the Johns Hopkins University DTI atlas in ICBM space (ICBM-DTI-81 white matter labels atlas) [39]. Additional image analysis details are available in the Supplementary section.

\section{STATISTICAL ANALYSIS}

Analysis 1: cortical thickness and white matter FA

We used a general linear model to compare both cortical thickness and white matter FA across diagnostic groups. Maps of $t$-statistics for group effects on cortical thickness at each vertex and FA at each voxel were then projected onto average templates, identifying areas where cortical thickness or FA were significantly different across groups. This statistical threshold was corrected for multiple comparisons using a $5 \%$ false discovery rate (FDR) correction for cortical thickness, and family-wise error rate (FWE) for FA. We further separated diagnostic groups according to age ("young" $<50$ years and "old" $\geq 50$ years). To test for differences in cortical thickness and FA, we used a two-tailed univariate F-test to compare cortical thickness and FA across all groups, with sex and parental education as covariates. This was followed by post hoc pairwise voxel-wise comparisons across groups. 
Table 1. Socio-demographic participant characteristics (sample 1)

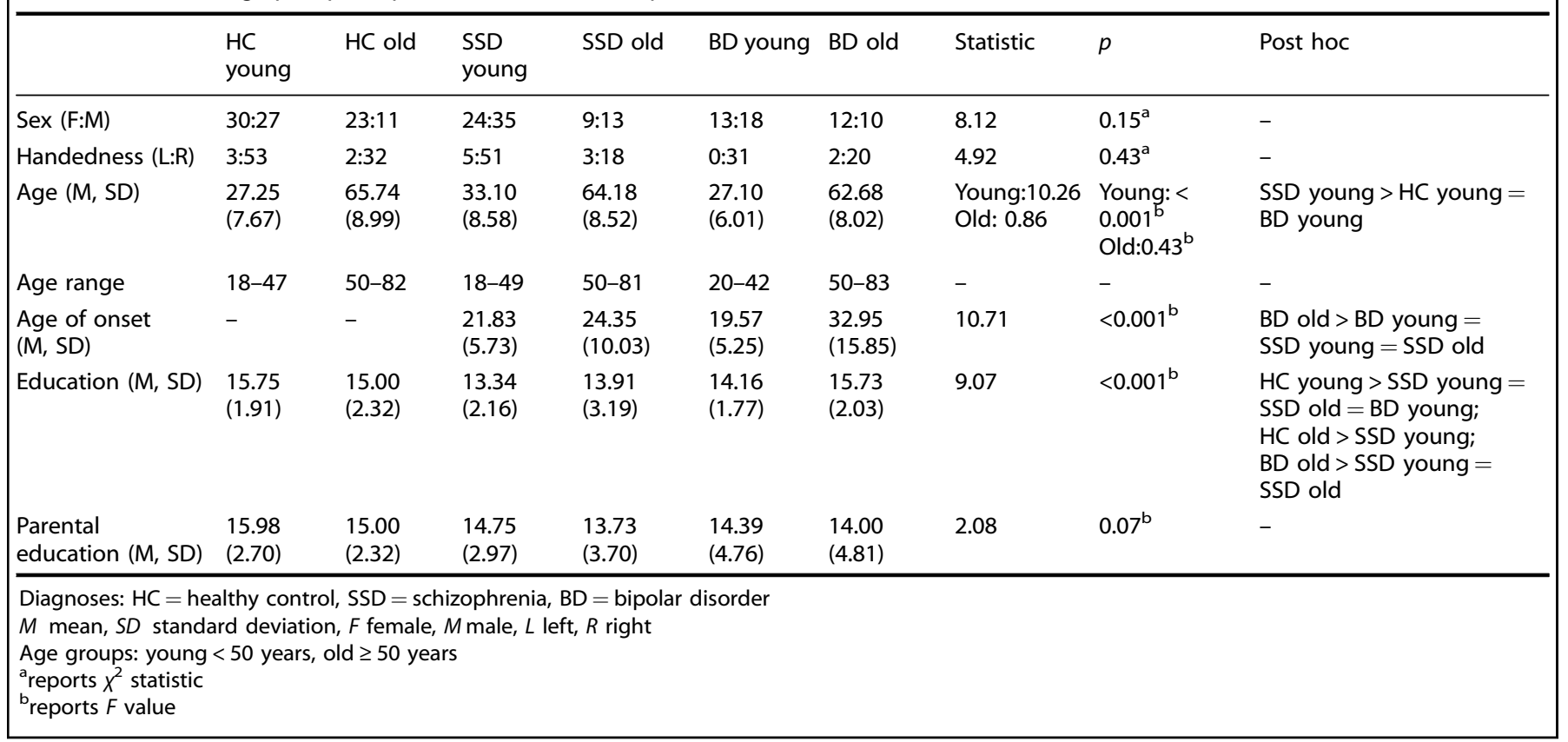

Analysis 2: association between cognitive performance and brain structure

Given the large body of evidence of one or two factors explaining the majority of the variance in cognitive function in healthy subjects [40-43] as well as in individuals with schizophrenia and $B D[21,22]$, we chose to perform principal component analysis (PCA) as a data reduction step to reduce multiple comparisons. We then used a general linear model to assess whether the principal component factor scores were associated with cortical thickness and/or FA, controlling for sex, age, and parental education in all participants collapsed together, as well as separately within each diagnosis. To correct for multiple comparisons, a 5\% FDR correction was used for cortical thickness and FWE was used for FA.

Analysis 3: predicted age versus chronological age

To predict age in the first sample using neuroimaging and neurocognitive performance measures, we trained a random forest algorithm [44] as implemented in the $R$ package randomForest (https://cran.r-project.org/web/packages/ randomForest/randomForest.pdf). The code can be found at https://github.com/navonacalarco/brainAge. We trained our model using $50 \mathrm{HCs}, 125$ features (cortical thickness in 78 regions, FA in 38 regions, and 9 cognitive performance scores), and a forest size of 10,000 trees. The model was tested on 175 participants $(41 \mathrm{HCs}$, 81 schizophrenia, $53 \mathrm{BD}$ ), none of whom overlapped with the training data set. The difference between predicted brain age and chronological age was calculated and was tested for significance using a two-tailed one-sample $t$-test. The age delta was compared across diagnostic categories using a two-tailed $F$-test with post hoc $t$-tests. We also used average mean squared error to determine which features were most important to prediction. We repeated this analysis in the second sample, using only neuroimaging features (cortical thickness in 78 regions and FA in 38 regions). The training dataset for the second sample was composed of $50 \mathrm{HCs}$, and the model was tested on 97 nonoverlapping participants ( $30 \mathrm{HCs}, 67$ schizophrenia). The healthy control group in both samples was randomly divided into the training and testing set. The training and test sets were examined for any difference in age, sex, or age range.

\section{RESULTS}

Participant characteristics

Socio-demographic characteristics of the first sample are shown in Table 1 (cognitive and clinical characteristics are shown in Supplementary Table 1 and Supplementary Table 2). There were significant differences between diagnostic groups in education $(F(2,221)=17.03, p<0.001)$, with $\mathrm{BD}$ and $\mathrm{HC}$ participants reporting higher education than those with schizophrenia; parental education $(F(2,168)=4.02, p=0.02)$, with HC participants reporting higher parental education than those with schizophrenia; and chlorpromazine-equivalent antipsychotic dose $(t(132)=-3.11, p=$ $0.002)$, with participants with schizophrenia more heavily medicated than those with BD. We assessed whether chlorpromazine equivalents were associated with cortical thickness or FA in participants with schizophrenia and BD: there were no significant associations after multiple comparison correction. In all, $64 \%$ of patients with BD had psychotic features when ill.

\section{Brain structure}

Cortical thickness. Cortical thickness differed between the six groups throughout the brain $(F(5,195)=24.12, p<0.001)$ (Fig. 1A), particularly in the frontal cortex and cortical midline regions. In post hoc pairwise comparisons (Fig. 2A), cortical thickness was significantly decreased in younger participants with schizophrenia compared to both younger $\mathrm{BD}(t(72)=6.86, p<0.001)$ and $\mathrm{HCs}$ $(t(100)=-3.90, p<0.001)$. No cortical thickness differences were found between HCs and BD. In older participants, few differences were found across diagnostic categories with small effect sizes, as seen in Fig. 2A. All reported results survived multiple comparison correction at the voxel level.

FA. FA differed among groups (Fig. 1B), mainly in the body $(F(5,173)=13.30, p<0.001)$ and splenium $(F(5,173)=13.28, p<$ $0.001)$ of the corpus callosum, left $(F(5,173)=12.54, p<0.001)$ and right $(F(5,173)=10.95, p<0.001)$ cerebral peduncles, left $(F(5,173)=$ $11.11, p<0.001)$ and right $(F(5,173)=12.86, p<0.001)$ superior corona radiata, and the left $(F(5,173)=8.09, p<0.001)$ and right $(F(5,173)=9.31, p<0.001)$ posterior limb of the internal capsule. Post hoc pairwise comparisons (Fig. $3 \mathrm{~A}$ ) showed significantly lower voxel-wise FA in younger participants with schizophrenia compared 
A

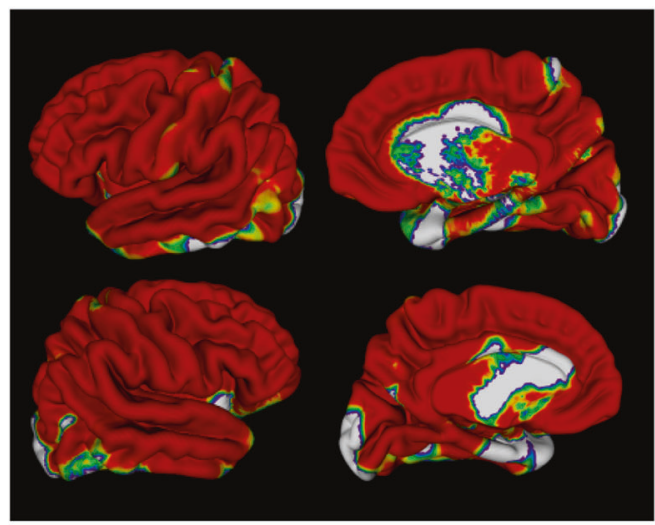

0.00

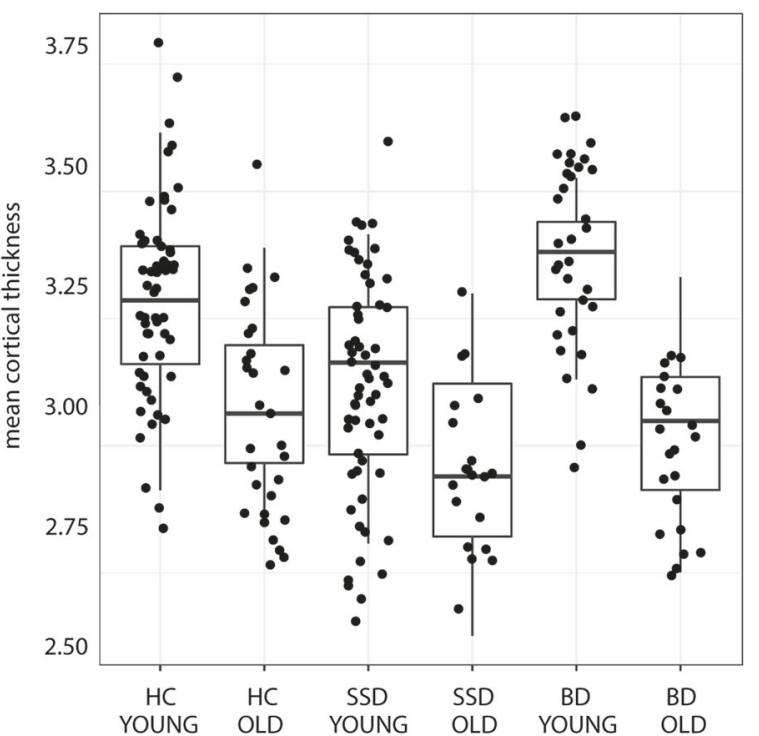

B

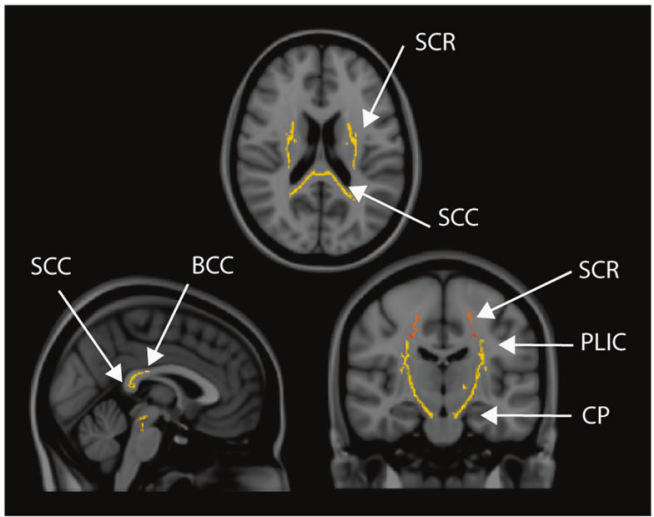

0.00

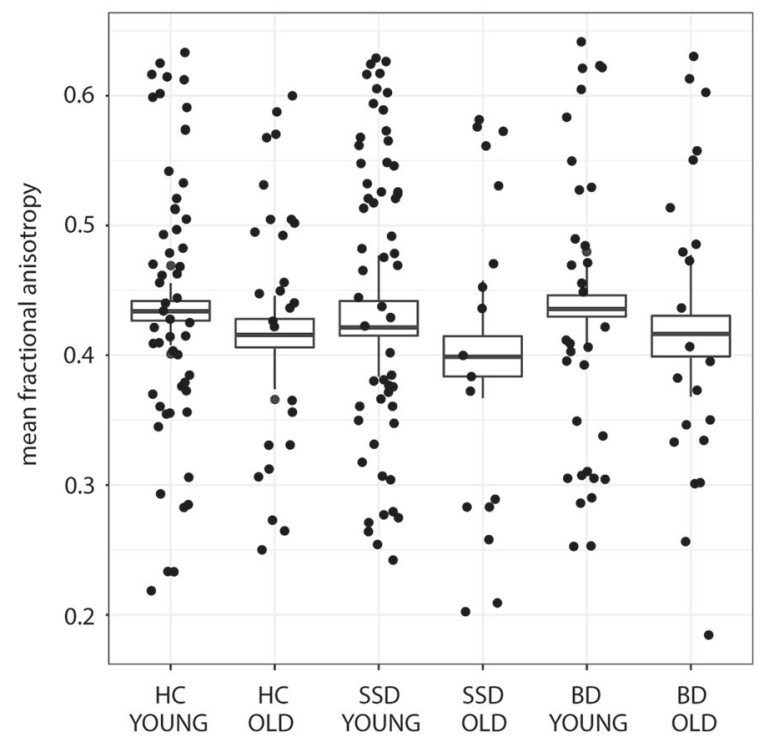

Fig. 1 Group differences in a cortical thickness and $\mathbf{b}$ fractional anisotropy, controlling for sex and parental education. a Mean brain-wide cortical thickness differences after controlling for sex and parental education, following an $F$-test $(F(5,195)=24.12, p<0.001)$, displayed by group, showing that older individuals have the lowest cortical thickness, followed by younger people with schizophrenia. $\mathbf{b}$ Mean differences in a number of white matter tracts, using $F A$ as the diffusion metric controlling for sex and parental education, following an $F$-test $(F(5,173)=$ $12.01, p<0.001)$, closely mirroring cortical thickness results. Unlike mean cortical thickness, no difference was seen in mean FA between SSD young and BD young participants. Legend. Diagnoses: $\mathrm{HC}=$ healthy control, $\mathrm{SSD}=\mathrm{schizophrenia}$ spectrum disorder, $\mathrm{BD}=$ bipolar disorder. White matter tracts: SCC = splenium of the corpus callosum, BCC = body of the corpus callosum, SCR $=$ superior corona radiata, $\mathrm{CP}=$ cerebral peduncles, $\mathrm{PLIC}=$ posterior limb of the internal capsule. Age groups: young $<50$ years, old $\geq 50$ years. The color scale shows (a) FDR-corrected and b FWE-corrected $p$ values $<0.05$

with both younger HCs and BD participants, but there were no differences between $\mathrm{HCs}$ and $\mathrm{BD}$. In older participants, there were few differences in white matter between diagnostic groups. All reported results survived multiple comparison correction at the voxel level. Detailed results for post hoc analyses are available in the Supplementary section.

\section{Brain structure and cognitive performance}

We included 214 subjects out of 225 in the PCA. Cognitive data were not available for the remaining 11 subjects. Our neurocognitive data loaded onto two significant factors, with the BACS Symbol Coding, CPT-IP, WMS-III: Spatial Span, Letter-Number Span, Letter-Number Span, HVLT-R, BVMT-R, and NAB Mazes, loading onto PC1 (43.87\% of variance explained), and the Trails Making Test: Part A and Trails Making Test: Part B loading onto PC2 (11.72\% of variance explained) (See Supplementary section for details, including Supplementary Figure 1).

In the entire sample, when controlling for sex, age, and parental education, we found that the PC1 cognitive score was significantly associated with cortical thickness in the inferior temporal gyrus in the right hemisphere $(r(189)=0.34, p<0.001)$, the left $(r(189)=$ $0.34, p<0.001)$ and right $(r(189)=0.33, p<0.001)$ superior frontal gyrus, left $(r(189)=0.28, p<0.001)$ and right $(r(189)=0.35, p<$ $0.001)$ inferior frontal gyrus, left $(r(189)=0.28, p<0.001)$ and right $(r(189)=0.29, p<0.001)$ superior temporal gyrus, left $(r(189)=$ $0.23, p<0.001)$ and right $(r(189)=0.28, p<0.001)$ middle temporal gyrus, and the left $(r(189)=0.18, p=0.007)$ and right $(r(189)=0.24, p<0.001)$ inferior parietal lobule (Fig. 2B). The PC1 score was also significantly and positively associated with FA in the splenium $(r(178)=0.30, p<0.001)$ and body of the corpus callosum $(r(178)=0.30, p<0.001)$ followed by the left $(r(178)=$ $0.24, p<0.001)$ and right $(r(178)=0.30, p<0.001)$ anterior corona radiata, left $(r(178)=0.28, p<0.001)$ and right $(r(178)=0.28, p<$ $0.001)$ anterior limb of the internal capsule, the fornix $(r(178)=$ $0.19, p=0.007)$, and the right $(r(178)=0.13, p=0.04)$ superior longitudinal fasciculus (Fig. $3 \mathrm{~B}$ ). When the relationships between the PC1 cognitive score and brain measures (cortical thickness and FA) were analyzed separately for each diagnostic group, no 
A

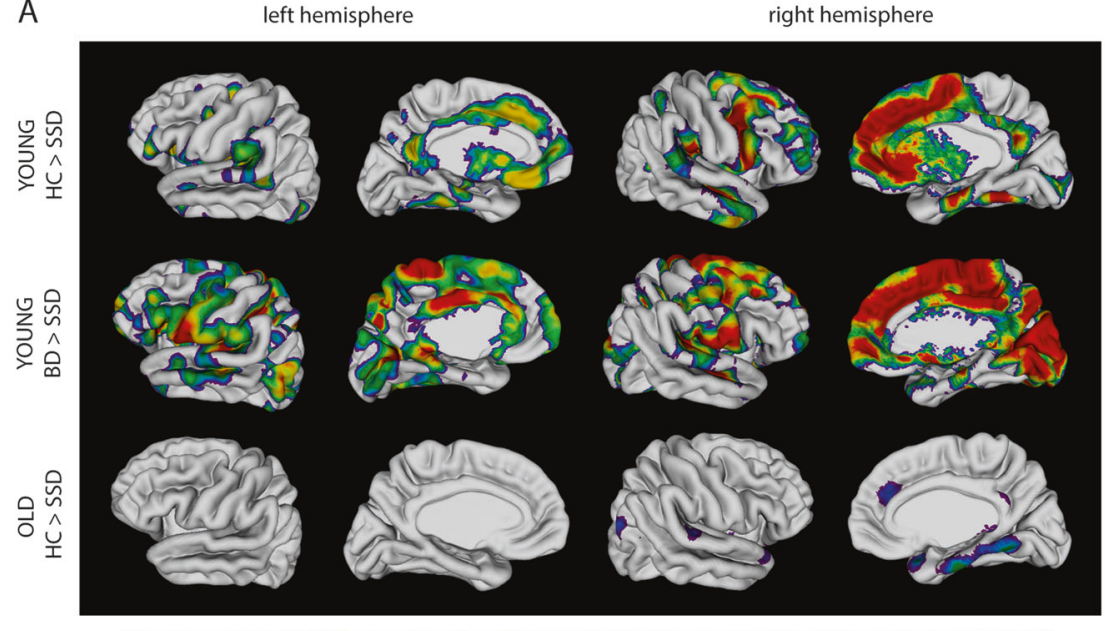

B

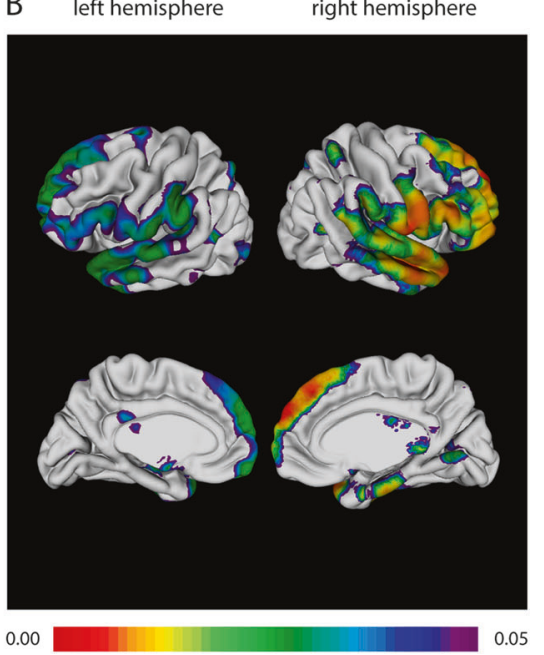

Fig. 2 a Significant post hoc differences in cortical thickness between diagnostic groups after controlling for sex and parental education. b Cortical thickness is positively associated with PC1 scores after controlling for sex, parental education, and age. a In younger subjects, schizophrenia participants show reduced cortical thickness compared with those with BD and HCs in fronto-temporal cortex, and cortical midline regions. In older subjects, schizophrenia participants show reduced cortical thickness compared to HCs again in fronto-temporal regions. Only significant results are shown: no differences between the HCs and BD groups were found. $\mathbf{b}$ The entire sample (comprising HCs and participants with schizophrenia or BD) was included in this analysis. Associations of cortical thickness with PC1 score from the neurocognitive battery demonstrating associations with superior and inferior frontal, superior, middle, and inferior temporal regions, and the inferior parietal lobule. Legend. Diagnoses: $\mathrm{HC}=$ healthy control, SSD = schizophrenia spectrum disorder, BD = bipolar disorder. Age groups: young $<50$ years, old $\geq 50$ years. The color scale shows FDR-corrected $p$ values $<0.05$

significant associations were observed. No associations between PC2 and brain measures were observed. All reported results survived multiple comparison correction at the voxel level.

\section{Predicted age versus chronological age}

In the first sample, the random forest analysis revealed that the delta of predicted and chronological age for HCs was 0.67 years (SE $= \pm$ 2.30), BD participants 0.14 years $(\mathrm{SE}= \pm 2.33)$, and schizophrenia participants 7.8 years ( $\mathrm{SE}= \pm 1.62$ ) (Fig. $4 \mathrm{~A})$. These deltas differed significantly across groups $(F(2,172)=9.94, p<0.001)$, with post hoc $t$-tests showing the schizophrenia group delta to be larger than that of HCs $(t(120)=-3.27, p=0.001)$ and $\operatorname{BD}(t(132)=-3.73, p<0.001)$. Interestingly, we found that HCs and BD participants were statistically equivalent in their predicted age. There was no significant difference $(t(51)=1.11, p=0.27)$ in age gap between $\mathrm{BD}$ patients who had psychosis when ill (delta $=0.76$ years, $\mathrm{SE}= \pm$ 1.06) compared with those who did not (delta $=-1.77$ years, $S E= \pm$ 2.41). Predicted and chronological age differed significantly in schizophrenia $(t(80)=4.98, p<0.001)$ but not in HCs $(t(40)=-0.35$, $p=0.73)$ and $\mathrm{BD}(t(52)=-0.13, p=0.90)$ participants. Regions most associated with age delta were cortical thickness in the bilateral Heschl's gyrus, left rolandic operculum, and left insula, and FA in the left anterior corona radiata and left posterior thalamic radiation. The algorithm performed similarly whether we included the nine cognitive scores or PC1 as training features.

In the second sample, results were generally congruent with those of the first sample (Fig. 4B; see Supplementary Table 3 for a summary of socio-demographic and clinical characteristics). Individuals with schizophrenia demonstrated a difference between predicted and chronological age of 6.12 years (SE $= \pm$ 1.99), whereas HCs showed a difference of only 1.80 years ( $S E= \pm$ 2.83), and these deltas were significantly different between groups $(t(95)=-2.83, p=0.005)$. Predicted and chronological age differed significantly in schizophrenia $(t(132)=-2.18, p=0.03)$ but not in $\mathrm{HCs}(t(54.19)=0.39, p=0.70)$. Although most of the top regions-of-interest identified in sample 1 were situated in the left temporal and insular cortices, in the second sample, regions identified as the most important for prediction were found in the frontal and parietal lobes of the left hemisphere: left paracentral lobule, left angular gyrus, left rectus gyrus, left middle frontal gyrus (orbital part), left stria terminalis of the fornix, left precuneus, and left superior frontal gyrus (medial orbital). No differences were observed between the training and test sets in age, sex, or age range in both samples.

\section{DISCUSSION}

We assessed the association of age, diagnosis, cognitive performance, and cortical thickness and white matter microstructure (FA) in younger ( $<50$ years) and older ( $\geq 50$ years) $\mathrm{HCs}$ and individuals with schizophrenia or $\mathrm{BD}$. We also tested whether brain age, compared with chronological age, is different amongst schizophrenia and BD groups compared with HCs. Overall, we found significant differences across the age and diagnostic groups. Young participants with schizophrenia showed decreased cortical thickness and FA compared with young HCs and BD; diagnostic group differences were much less prominent in older individuals. This suggests that reductions in cortical thickness in brain structure occur early in individuals with schizophrenia. We also found an association between brain structure and cognitive performance independent of diagnosis, which supports the relevance of brain structure alterations for functionality. Finally, we found that predicted brain age, in comparison to chronological age, was approximately 8 years greater in participants with schizophrenia, supporting the hypothesis of early brain aging in individuals with schizophrenia, but not BD.

We view early hit non-progressive and accelerated aging mechanisms as inter-related, because our data suggest that the "accelerated aging" occurs very early in the disease course and then aging (or age-related changes) seem relatively equivalent whether in $\mathrm{HCs}$ or in those with severe mental illness (i.e., schizophrenia and BD). Therefore, the hit is early, and we believe that the accelerated aging likely occurs during that period, resulting in deterioration of brain structure and cognitive performance. However, during the remainder of the lifespan, it appears that the disease is non-progressive. 
A

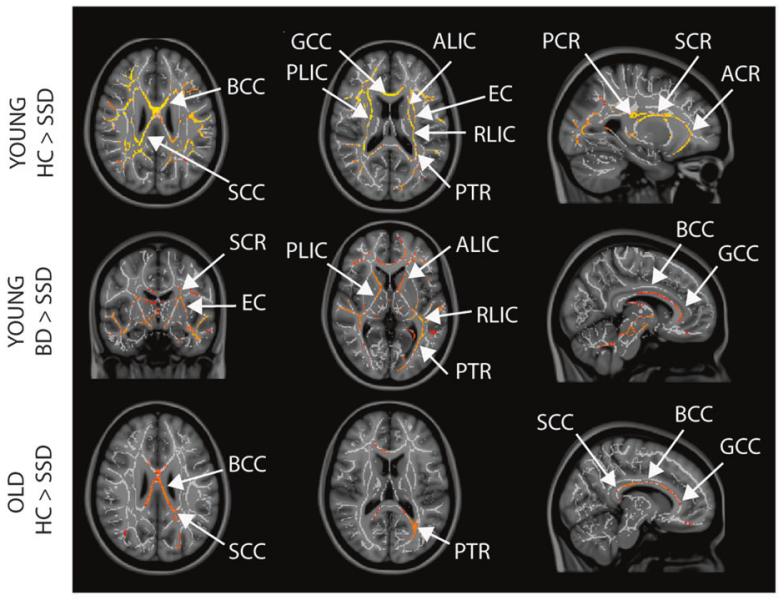

B

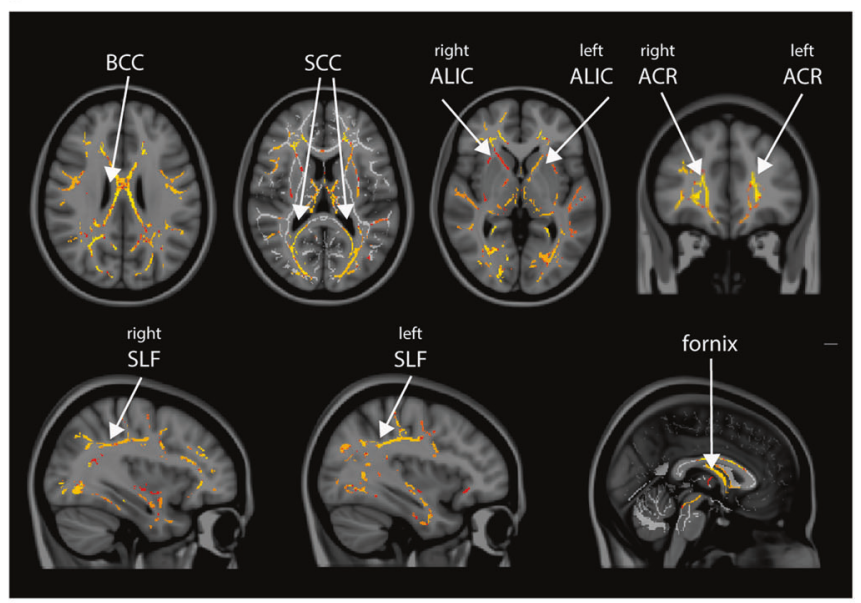

0.00

0.00

Fig. 3 a Significant post hoc differences in white matter FA between diagnostic groups after controlling for sex and parental education. $\mathbf{b}$ FA of the white matter tracts are positively associated with PC1 scores, after controlling for sex, parental education, and age. a The group-wise pattern in white matter FA comparisons is similar to cortical thickness comparisons shown in $2 \mathrm{~A}$. Only significant results are shown: no differences between $\mathrm{HCs}$ and participants with BD were found. $\mathbf{b}$ The entire sample (comprising $\mathrm{HCs}$ and participants with schizophrenia or BD) was included in this analysis. Associations of white matter FA with PC1 score demonstrating relationships of cognitive scores with FA of key fronto-temporal (e.g., superior longitudinal fasciculus) and interhemispheric (e.g., corpus callosum) tracts. Legend: Diagnoses: HC $=$ healthy control, SSD = schizophrenia spectrum disorder, BD = bipolar disorder. White matter tracts: $F A=$ fractional anisotropy, GCC $=$ genu of the corpus callosum, BCC = body of the corpus callosum, SCC = splenium of the corpus callosum, ALIC = anterior limb of the internal capsule, $\mathrm{PLIC}=$ posterior limb of the internal capsule, $\mathrm{RLIC}=$ retrolenticular limb of internal capsule, $\mathrm{ACR}=$ anterior corona radiata, $\mathrm{PCR}=$ posterior corona radiata, $\mathrm{SCR}=$ superior corona radiata, $\mathrm{SLF}=$ superior longitudinal fasciculus, $\mathrm{EC}=$ external capsule, PTR $=$ posterior thalamic radiation. Age groups: young $<50$ years, old $\geq 50$ years. The color scale shows FWE-corrected $p$ values $<0.05$

Differences in cortical thickness and FA associated with age and diagnosis support and extend the results of a series of previous studies that found decreases in cortical thickness and FA with age $[9,10,12]$ and in individuals with schizophrenia and BD compared with $\mathrm{HCs}[8,12,19]$. Brain structure alterations observed in the present study were particularly prominent in fronto-temporal regions, as found in previous studies [11]. Furthermore, we replicated some of our previous findings showing decreased FA in specific white matter tracts in younger individuals with schizophrenia compared with $\mathrm{HCs}$, but minimal differences in older groups [12]. A recent study showed rapid gray matter loss in schizophrenia before the age of 45 , with a steady decline in white matter FA after the age of 35 [45]. Although our cortical thickness results support this finding (greater difference in the young group compared with the old group), our FA results contradict the findings of this study (greater difference in the young group compared with the old group). This discrepancy in findings may be explained by the difference in the age cutoffs for gray matter and white matter changes. The age when gray matter changes stabilize is much closer to our age group cutoff of 50 years. In addition, Cropley and colleagues (2017) did not observe any participants $>65$ years of age. Our current results extend prior work by including participants throughout the entire adult lifespan (i.e., age 18 to mid 80 s) and by including HCs and people with schizophrenia and BD. Taken together, these and our prior results suggest that schizophrenia is associated with alterations in both cortical thickness and FA, and that BD brain structure, although perhaps intermediate between HCs and schizophrenia, is closer to HCs than schizophrenia. Cortical thickness and white matter FA were significantly lower in participants with schizophrenia compared with BD, but contrary to previous studies $[8,46]$, we did not find significant differences in brain structure between $\mathrm{HCs}$ and individuals with $\mathrm{BD}$ and thus our results do not support the presence of accelerated aging in the brain of participants with $\mathrm{BD}$. This may be owing to the fact that our BD participants were euthymic at the time of scanning and thus are treatment responsive.
Regarding cognitive performance, our results suggest that relationships between brain structure and cognitive performance are independent of diagnosis. The results of previous studies are mixed, with some studies finding brain-cognition associations to differ across diagnostic groups [47] and others finding similar associations across groups [48]. Inconsistencies may be owing to the specific measures of cognitive function and brain structure used in different studies. If we had looked at each cognitive performance score separately (instead of performing a principal component analysis), we may have found differences across groups in regard to specific cognitive domains.

Our finding that the brains of individuals with schizophrenia appear over 8 years older than their chronological age is quite striking, and is greater than the extent of accelerated aging reported in the majority of studies in the literature. Using support vector regression, Koutsouleris et al. [49] compared people with schizophrenia, major depressive disorder, and borderline personality disorder, with high-risk individuals, and found that those with schizophrenia demonstrated the greatest brain age compared with the other groups, with an increase in brain age of 5.5 years in schizophrenia. Using the same support vector regression method, Schnack and colleagues [50] found that individuals with schizophrenia demonstrated an average difference of 5.5 years between brain and chronological age. Using relevance vector regression, Nenadić and colleagues [51] found that individuals with schizophrenia demonstrated an increased brain age of 2.56 years compared with $\mathrm{HCs}$ and bipolar I, and there was no difference between $\mathrm{HCs}$ and $\mathrm{BD}$. Another recent study used gradient boosting machines and showed a 3.9 year gap between brain age and chronological age in individuals with schizophrenia and a gap of 2 years in those with BD [52]. Similarly, Hajek et al. [53] used relevance vector regression and showed an increased brain age of 8.79 years in first-episode schizophrenia compared with $\mathrm{HCs}$, and no difference between $\mathrm{HCs}$ and BD. Our finding of an 8-year acceleration in schizophrenia may be attributable to an improved model deriving from a large age range (18-83 years), or that our 
A

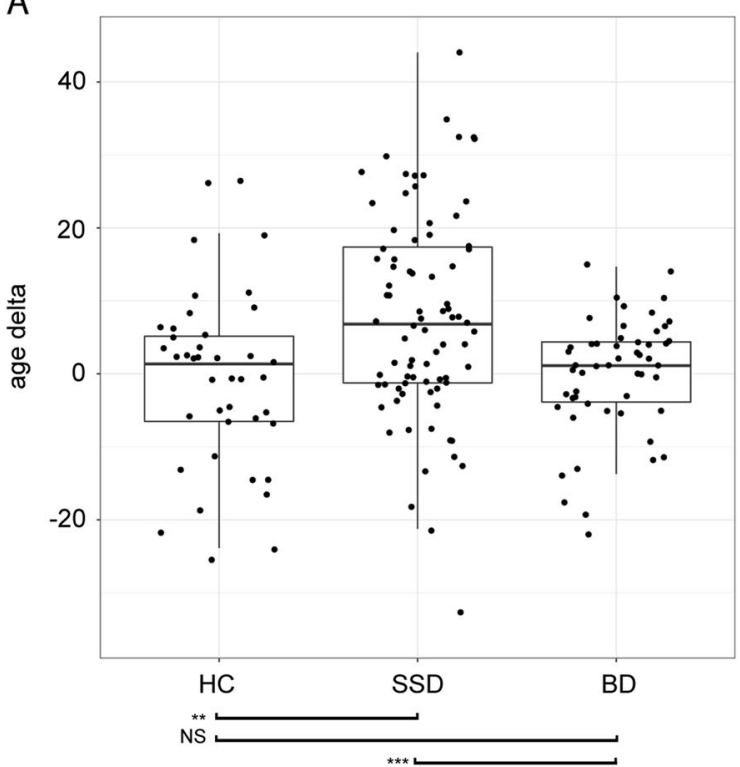

B

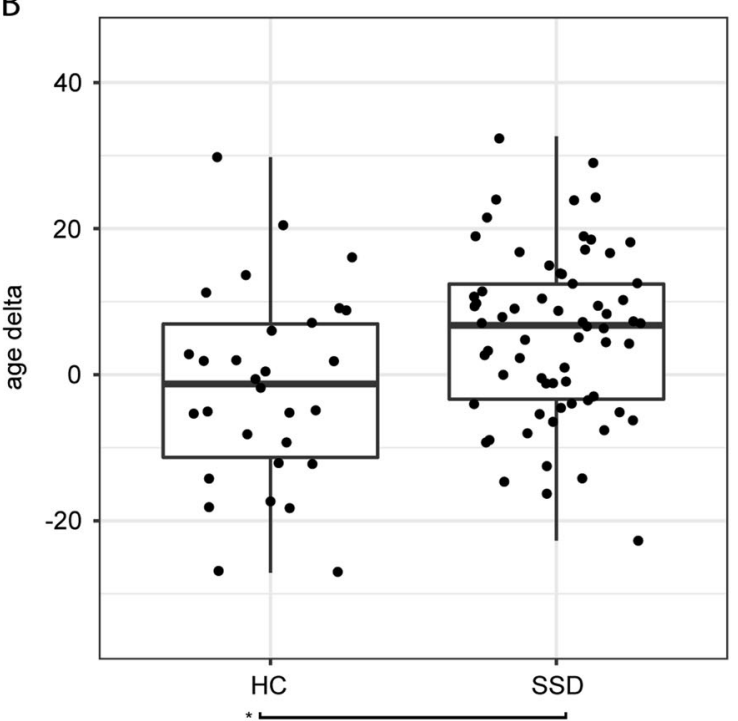

Fig. 4 Difference in predicted age and chronological age (age delta) in a sample 1 and $\mathbf{b}$ sample 2. Random forest analysis estimates higher delta in chronological versus predicted brain age for participants with schizophrenia. a In the first sample $(N=175 ; 41 \mathrm{HC}, 81 \mathrm{SSD}, 53 \mathrm{BD})$, a model was trained on 78 cortical thickness features, 38 fractional anisotropy features, and 9 cognitive performance features from 50 nonoverlapping HCs. The schizophrenia group brain age delta was 7.8 years. Predicted and chronological age differed significantly in schizophrenia $(t(80)=4.98, p<0.001)$ but not in $\mathrm{HCs}(t(40)=-0.35, p=0.73)$ and $\mathrm{BD}(t(52)=-0.13, p=0.90)$ participants. Age deltas differed significantly across groups $(F(2,172)=9.94, p<0.001)$, with post hoc $t$-tests showing the schizophrenia group delta to be larger than that of HCs $(t(120)=-3.27, p=0.001)$ and BD $(t(132)=-3.73, p<0.001)$. HCs and BD participants were statistically equivalent in their predicted age. b In the second sample $(N=122 ; 55 \mathrm{HC}, 67 \mathrm{SSD})$ using a model trained on the same 78 cortical thickness features and 38 fractional anisotropy features from 25 non-overlapping $\mathrm{HCs}$, the schizophrenia group brain age delta was 8.20 years. Predicted and chronological age differed significantly in schizophrenia $(t(65)=5.33, p<0.001)$ but not in $\mathrm{HCs}(t(53)=1.59, p=0.12)$. Age delta was significantly higher $(t(117.45)=$ $-2.56, p=0.01$ ) in the SSD group compared with HC. Legend. Diagnoses: $\mathrm{HC}=$ healthy control, SSD = schizophrenia spectrum disorder, $\mathrm{BD}=$ bipolar disorder. Significance codes: NS = not significant, ${ }^{*} p<.05,{ }^{* *} p<.01,{ }^{* * *} p<.001$

features included both gray matter and white matter measurements as well as cognitive performance scores. Overall, our study and prior studies suggest that brain age differs from chronological age, and that difference may be more marked in people with severe mental illness. Given that recent research also suggests that older brain-predicted age in healthy individuals is associated with behaviors and physiological changes that are observed with typical aging [54], this marked difference in brain age and chronological age may represent a feature of mental illness and constitute a potential target for treatment.

Our findings should be considered in light of some limitations. First, although our overall sample was reasonably large for a neuroimaging study, the size of groups categorized by diagnosis and age were small, and this may have precluded detection of some associations. The sample size was larger for the younger diagnostic groups compared with all three older groups. Thus, the power to detect a difference among the younger groups was greater than that in the older groups, which may explain why we observed fewer statistically significant differences among the older diagnostic groups. Second, though we assessed if brain structure was associated with current medication exposure, it would have been more precise to consider the cumulative exposure of antipsychotics. Longitudinal prospective studies following individuals from their first episode of psychosis through the progression of their illness will be necessary to address this question with confidence. In addition, as global thickness has been shown to be related to cognitive performance [55], assessing the relationship between relative thickness and cognitive performance will help further elucidate the relationship between cortical thickness deficits and cognition in schizophrenia and BD.

We have previously examined younger and older groups of $\mathrm{HC}$ and people with schizophrenia [56-58]. We have also examined people with BD using 50 as the age cutoff [59]. In other studies, we have modeled age as a continuous variable [60]. An age cutoff into two groups provides a simpler heuristic, and leads us to conclusions consistent with our prior work, i.e., that differences are most apparent earlier in the lifespan, and less apparent later in the lifespan. Similarly age-related brain change appears to occur early in mental illness, and does not seem to be occurring in later-life in a manner more prominent than that seen in HC. In summary, our results support an "early hit", neurodevelopmental, nonprogressive mechanism for both schizophrenia and BD, and a mechanism of early aging in schizophrenia, but not to the same degree in BD. Future studies will be necessary to assess potential mediators of this early aging and identify specific causative mechanisms.

\section{FUNDING AND DISCLOSURE}

This work was supported by funding from the Canadian Institutes of Health Research (ANV and BHM). ANV currently receives funding from the National Institute of Mental Health, Canadian Institutes of Health Research; Canada Foundation for Innovation; Ontario Ministry of Research and Innovation, CAMH Foundation, and University of Toronto. BHM currently receives research funding from Brain Canada; the Centre for Addiction and Mental Health Foundation; the Canadian Institutes of Health Research; Patient-Centred Outcomes Research Institute (PCORI) and the US National Institutes of Health (NIH). ALW receives funding from the Natural Sciences and Engineering Research Council of Canada and the SickKids Foundation. GF has received funding from the Canadian Institutes of Health Research, the Ontario Mental Health Foundation, the Brain and Behaviour Research Foundation, the Centre for Addiction and Mental Health Foundation and the Canadian Foundation for Innovation, has served on advisory boards for Roche and Takeda, and received speaker fees from Roche, Novartis, and Lundbeck. TKR has received research support from Brain Canada; Brain and Behavior Research Foundation; BrightFocus Foundation; Canada Foundation for Innovation; Canada Research Chair; Canadian Institutes of Health 
Research; Centre for Aging and Brain Health Innovation; National Institutes of Health; Ontario Ministry of Health and Long-Term Care; Ontario Ministry of Research and Innovation; and the Weston Brain Institute. The remaining authors have nothing to disclose.

\section{ADDITIONAL INFORMATION}

Supplementary Information accompanies this paper at (https://doi.org/10.1038/ s41386-018-0298-z)

Publisher's note: Springer Nature remains neutral with regard to jurisdictional claims in published maps and institutional affiliations.

\section{REFERENCES}

1. Kirkpatrick B, Messias E, Harvey PD, Fernandez-Egea E, Bowie CR. Is schizophrenia a syndrome of accelerated aging? Schizophr Bull. 2008;34:1024-32.

2. Okusaga OO. Accelerated aging in schizophrenia patients: the potential role of oxidative stress. Aging Dis. 2014;5:256-62.

3. Kirkpatrick B, Kennedy BK. Accelerated aging in schizophrenia and related disorders: future research. Schizophr Res. 2018;196:4-8.

4. Jin H, Folsom D, Sasaki A, Mudaliar S, Henry R, Torres M, et al. Increased Framingham 10-year risk of coronary heart disease in middle-aged and older patients with psychotic symptoms. Schizophr Res. 2011;125:295-9.

5. Rizzo LB, Costa LG, Mansur RB, Swardfager W, Belangero SI, Grassi-Oliveira R, et al. The theory of bipolar disorder as an illness of accelerated aging: implications for clinical care and research. Neurosci Biobehav Rev. 2014;42:157-69.

6. Lee A, Ratnarajah N, Tuan TA, Chen SH, Qiu A. Adaptation of brain functional and structural networks in aging. PLoS ONE 2015;10:e0123462.

7. Frangou S. A systems neuroscience perspective of schizophrenia and bipolar disorder. Schizophr Bull. 2014;40:523-31.

8. Oertel-Knochel V, Reuter J, Reinke B, Marbach K, Feddern R, Alves G, et al. Association between age of disease-onset, cognitive performance and cortical thickness in bipolar disorders. J Affect Disord. 2015;174:627-35.

9. Thormodsen R, Rimol LM, Tamnes CK, Juuhl-Langseth M, Holmen A, Emblem KE, et al. Age-related cortical thickness differences in adolescents with early-onset schizophrenia compared with healthy adolescents. Psychiatry Res. 2013; 214:190-6.

10. Storsve AB, Fjell AM, Tamnes CK, Westlye LT, Overbye K, Aasland HW, et al. Differential longitudinal changes in cortical thickness, surface area and volume across the adult life span: regions of accelerating and decelerating change. J Neurosci. 2014;34:8488-98.

11. van Haren NE, Schnack HG, Cahn W, van den Heuvel MP, Lepage C, Collins L, et al. Changes in cortical thickness during the course of illness in schizophrenia. Arch Gen Psychiatry. 2011;68:871-80.

12. Voineskos AN, Lobaugh NJ, Bouix S, Rajji TK, Miranda D, Kennedy JL, et al. Diffusion tensor tractography findings in schizophrenia across the adult lifespan. Brain. 2010;133:1494-504.

13. Voineskos AN, Rajji TK, Lobaugh NJ, Miranda D, Shenton ME, Kennedy JL, et al. Age-related decline in white matter tract integrity and cognitive performance: a DTI tractography and structural equation modeling study. Neurobiol Aging. 2012;33:21-34.

14. Sullivan EV, Rohlfing T, Pfefferbaum A. Quantitative fiber tracking of lateral and interhemispheric white matter systems in normal aging: relations to timed performance. Neurobiol Aging. 2010;31:464-81.

15. Kochunov P, Glahn DC, Rowland LM, Olvera RL, Winkler A, Yang YH, et al. Testing the hypothesis of accelerated cerebral white matter aging in schizophrenia and major depression. Biol Psychiatry. 2013;73:482-91.

16. Nazeri A, Chakravarty MM, Rajji TK, Felsky D, Rotenberg DJ, Mason M, et al. Superficial white matter as a novel substrate of age-related cognitive decline. Neurobiol Aging. 2015;36:2094-106.

17. Poletti S, Bollettini I, Mazza E, Locatelli C, Radaelli D, Vai B, et al. Cognitive performances associate with measures of white matter integrity in bipolar disorder. J Affect Disord. 2015;174:342-52.

18. Nilsson J, Thomas AJ, O'Brien JT, Gallagher P. White matter and cognitive decline in aging: a focus on processing speed and variability. J Int Neuropsychol Soc. 2014;20:262-7.

19. Ahmed M, Cannon DM, Scanlon C, Holleran L, Schmidt H, McFarland J, et al Progressive brain atrophy and cortical thinning in schizophrenia after commencing clozapine treatment. Neuropsychopharmacology. 2015;40: 2409-17.

20. Jeste DV, Wolkowitz OM, Palmer BW. Divergent trajectories of physical, cognitive, and psychosocial aging in schizophrenia. Schizophr Bull. 2011;37:451-5.
21. Keefe RSE, Bilder RM, Harvey PD, Davis SM, Palmer BW, Gold JM, et al. Baseline neurocognitive deficits in the CATIE schizophrenia trial. Neuropsychopharmacology. 2006;31:2033-46.

22. Harvey PD, Aslan $M$, Du $M$, Zhao $H$, Siever $\amalg$, Pulver $A$, et al. Factor structure of cognition and functional capacity in two studies of schizophrenia and bipolar disorder: Implications for genomic studies. Neuropsychology. 2016; 30:28-39.

23. Daban C, Martinez-Aran A, Torrent C, Tabarés-Seisdedos R, Balanzá-Martínez V, Salazar-Fraile, J, et al. Specificity of cognitive deficits in bipolar disorder versus schizophrenia. Psychotherapy and psychosomatics. 2006;75:72-84.

24. Krabbendam L, Arts B, van Os J, \& Aleman A. Cognitive functioning in patients with schizophrenia and bipolar disorder: a quantitative review. Schizophr. Res. 2005;80:137-149.

25. Wechsler D. Wechsler Test of Adult Reading (WTAR). Toronto, Ontario, Canada: Pearson Canada Assessment; 2001.

26. Hamilton M. Development of a rating scale for primary depressive illness. Br J Soc Clin Psychol. 1967;6:278-96.

27. Young RC, Biggs JT, Ziegler VE, Meyer DA. A rating scale for mania: reliability, validity and sensitivity. Br J Psychiatry. 1978;133:429-35.

28. First FirstM, Michael B, Spitzer RL, Miriam gibbon, Williams JBW. Structured Clinical Interview for DSM-IV Axis I Disorders. Clinician Version. Washington, DC: American Psychiatric Press, Inc; 1996.

29. Kay SR, Fiszbein A, Opler LA. The positive and negative syndrome scale (PANSS) for schizophrenia. Schizophr Bull. 1987;13:261-76.

30. Overall JE, Gorham DR. The brief psychiatric rating scale. Psychol Rep 1962;10:799-812

31. Andreasen NC Scale for the Assessment of Negative Symptoms [Internet]. PsyCTESTS Dataset. 1982. Available at https://doi.org/10.1037/t12696-000.

32. Kern RS, Nuechterlein KH, Green MF, Baade LE, Fenton WS, Gold JM, et al. The MATRICS Consensus Cognitive Battery, part 2: co-norming and standardization. Am J Psychiatry. 2008;165:214-20.

33. Nuechterlein KH, Green MF, Kern RS, Baade LE, Barch DM, Cohen JD, et al. The MATRICS Consensus Cognitive Battery, part 1: test selection, reliability, and validity. Am J Psychiatry. 2008;165:203-13.

34. Reitan RM, Wolfson D. The Halstead-Reitan Neuropsychological Battery. In: Goldstein G., Incagnoli T.M. (eds) Contemporary Approaches to Neuropsychological Assessment. Critical Issues in Neuropsychology. Springer, Boston, MA; 1985.

35. Lerch JP, Worsley K, Shaw WP, Greenstein DK, Lenroot RK, Giedd J, et al. Mapping anatomical correlations across cerebral cortex (MACACC) using cortical thickness from MRI. Neuroimage. 2006;31:993-1003.

36. Winkler AM, Ridgway GR, Webster MA, Smith SM, Nichols TE. Permutation inference for the general linear model. Neuroimage. 2014;92:381-97.

37. Tzourio-Mazoyer N, Landeau B, Papathanassiou D, Crivello F, Etard O, Delcroix N, et al. Automated anatomical labeling of activations in SPM using a macroscopic anatomical parcellation of the MNI MRI single-subject brain. Neuroimage. 2002;15:273-89.

38. Jahanshad N, Kochunov PV, Sprooten E, Mandl RC, Nichols TE, Almasy L, et al. Multi-site genetic analysis of diffusion images and voxelwise heritability analysis: a pilot project of the ENIGMA-DTI working group. Neuroimage. 2013;81:455-69.

39. Mori S, Wakana S, Nagae-Poetscher LM, van Zijl P. MRI atlas of human white matter. Am Soc Neuroradiol. 2006;27:1384.

40. Carroll JB. Human cognitive abilities: a survey of factor-analytic studies. Cambridge University Press; 1993.

41. Jensen AR. The $g$ factor: the science of mental ability. JSTOR; Westport, CT: Greenwood, 1998.

42. Penke L, Maniega SM, Bastin ME, Valdés Hernández MC, Murray C, Royle NA, et al Brain white matter tract integrity as a neural foundation for general intelligence. Mol Psychiatry. 2012;17:1026-30.

43. Penke L, Muñoz Maniega S, Murray C, Gow AJ, Hernández MCV, Clayden JD, et al. A general factor of brain white matter integrity predicts information processing speed in healthy older people. J Neurosci. 2010;30:7569-74.

44. Breiman L. Random forests. Mach Learn. 2001;45:5-32.

45. Cropley VL, Klauser P, Lenroot RK, Bruggemann J, Sundram S, Bousman C, et al. Accelerated gray and white matter deterioration with age in schizophrenia. Am J Psychiatry. 2017; 174:286-95.

46. Sussmann JE, Lymer GK, McKirdy J, Moorhead TW, Munoz Maniega S, Job D, et al. White matter abnormalities in bipolar disorder and schizophrenia detected using diffusion tensor magnetic resonance imaging. Bipolar Disord. 2009;11:11-18.

47. McKenna BS, Theilmann RJ, Sutherland AN, Eyler LT. Fusing functional MRI and diffusion tensor imaging measures of brain function and structure to predict working memory and processing speed performance among inter-episode bipolar patients. J Int Neuropsychol Soc. 2015;21:330-41.

48. Fears SC, Schur R, Sjouwerman R, Service SK, Araya C, Araya X, et al. Brain structure-function associations in multi-generational families genetically enriched for bipolar disorder. Brain. 2015;138:2087-102. 
Brain structure, cognition, and brain age in schizophrenia, bipolar...

S Shahab et al.

906

49. Koutsouleris N, Davatzikos C, Borgwardt S, Gaser C, Bottlender R, Frodl T, et al. Accelerated brain aging in schizophrenia and beyond: a neuroanatomical marker of psychiatric disorders. Schizophr Bull. 2014;40:1140-53.

50. Schnack HG, van Haren NEM, Nieuwenhuis M, Hulshoff Pol HE, Cahn W, Kahn RS. Accelerated brain aging in schizophrenia: a longitudinal pattern recognition study. Am J Psychiatry. 2016;173:607-16.

51. Nenadić I, Dietzek M, Langbein K, Sauer H, Gaser C. BrainAGE score indicates accelerated brain aging in schizophrenia, but not bipolar disorder. Psychiatry Res. 2017;266:86-89.

52. Kaufmann T, van der Meer D, Doan NT, Schwarz E, Lund MJ, Agartz I, et al. Genetics of brain age suggest an overlap with common brain disorders. 2018; https://doi.org/10.1101/303164.

53. Hajek T, Franke K, Kolenic M, Capkova J, Matejka M, Propper L, et al. Brain ae in early stages of bipolar disorders or schizophrenia. Schizophr Bull. 2017; https:// doi.org/10.1093/schbul/sbx172.

54. Cole JH, Ritchie SJ, Bastin ME, Valdés Hernández MC, Muñoz Maniega S, Royle N, et al. Brain age predicts mortality. Mol Psychiatry. 2017; 23:1385-92.

55. Vuoksimaa E, Panizzon MS, Chen C-H, Fiecas M, Eyler LT, Fennema-Notestine C, et al. Is bigger always better? The importance of cortical configuration with respect to cognitive ability. Neuroimage. 2016;129:356-66.
56. Rajji TK, Voineskos AN, Butters MA, Miranda D, Arenovich T, Menon M, et al. Cognitive performance of individuals with schizophrenia across seven decades: a study using the MATRICS consensus cognitive battery. The American Journal of Geriatric Psychiatry. 2013;21:108-18.

57. Kumar S, Mulsant BH, Tsoutsoulas C, Ghazala Z, Voineskos AN, Bowie CR, et al. An optimal combination of MCCB and CANTAB to assess functional capacity in older individuals with schizophrenia. International journal of geriatric psychiatry. 2016;31:1116-23.

58. Tsoutsoulas C, Mulsant BH, Kumar S, Ghazala Z, Voineskos AN, Menon M, et al. Anticholinergic Burden and Cognition in Older Patients With Schizophrenia. The Journal of clinical psychiatry. 2017;78:e1284-90.

59. Vaccarino SR, Rajji TK, Gildengers AG, Waters SE, Butters MA, Menon M, et al. Allostatic load but not medical burden predicts memory performance in latelife bipolar disorder. International journal of geriatric psychiatry. 2018;33: 546-52.

60. Kalache SM, Mulsant BH, Davies SJ, Liu AY, Voineskos AN, Butters MA, et al. The impact of aging, cognition, and symptoms on functional competence in individuals with schizophrenia across the lifespan. Schizophrenia bulletin, 2014;41:374-81. 